\title{
Exploring differences in prevalence of diagnosed, measured and undiagnosed hypertension: the case of Ireland and the United States of America
}

\author{
Irene Mosca $\cdot$ Rose Anne Kenny
}

Received: 25 September 2013/Revised: 9 May 2014 / Accepted: 2 June 2014 / Published online: 19 June 2014

(C) Swiss School of Public Health 2014

\begin{abstract}
Objectives To: (1) compare prevalence of diagnosed, measured, total and undiagnosed hypertension among late middle-aged adults in the United States of America and Ireland; (2) identify the most important predictors of hypertension and compare them across the two countries; (3) investigate whether cross-national differences in prevalence rates are explained by country differences in behavioural risk factors.

Methods We use data from the 2008 and 2010 waves of the Health and Retirement Study $(n=1,938)$ and the first wave $(2009 / 2011)$ of The Irish Longitudinal Study on Ageing $(n=1,455)$. We employ probit models to determine whether individual attributes, socioeconomic characteristics and behavioural health factors, including smoking, alcohol consumption and obesity, are associated with hypertension. We do not consider whether respondents are on antihypertensive medication.

Results Prevalence of diagnosed hypertension is higher in the United States of America (48.6 versus $32.4 \%$ ).
\end{abstract}

I. Mosca $(\bowtie) \cdot$ R. A. Kenny

The Irish Longitudinal Study on Ageing (TILDA),

Department of Medical Gerontology, Lincoln Gate,

Trinity College, Dublin, Ireland

e-mail: moscai@tcd.ie

I. Mosca

Economic and Social Research Institute (ESRI), Dublin, Ireland

\section{R. A. Kenny}

Mercer's Institute for Successful Ageing,

St. James's Hospital, Dublin, Ireland

R. A. Kenny

Trinity College Institute of Neuroscience, Trinity College,

Dublin, Ireland
Prevalence of undiagnosed hypertension is higher in Ireland (41.2 versus $19.7 \%$ ). Little of the difference in prevalence rates is explained by country differences in behavioural risk factors.

Conclusions A greater focus on prevention of high blood pressure is necessary in Ireland.

Keywords Hypertension - Diagnosed · Measured · Undiagnosed · Ireland · USA

\section{Introduction}

Hypertension, also known as high blood pressure, is one of the most common chronic conditions worldwide. Even more important, hypertension is an important risk factor for more serious conditions that carry greater risk of disability and death, primarily cardio- and cerebro-vascular events, and is the single most important modifiable risk factor for stroke and myocardial infarction in both developed and developing countries (Kearney et al. 2005). Hypertension already affects one billion people worldwide and is a global health issue (WHO 2013).

In many population studies with a focus or a component on health, hypertension prevalence rates are derived from 'self-reports': respondents are asked to report whether they have hypertension at present and/or have ever been diagnosed with hypertension. Prevalence based on self-reports might, however, seriously underestimate the actual prevalence. This is because moderate, and even high levels of high blood pressure are typically asymptomatic and rates of undiagnosed or undetected hypertension are generally high (WHO 2013).

A way to assess the extent to which hypertension is undiagnosed is to collect both self-reported and objective 
measures for the same respondents. This is, however, rarely done in population studies. Also, international comparisons of prevalence rates are difficult, as surveys often differ in their methodology and design and survey data for hypertension are difficult to standardize.

Few studies have compared prevalence rates among middle-aged and older adults across Europe and the United States of America (USA). In a widely cited paper, Banks et al. (2006) found that prevalence of both measured and self-reported hypertension among middle-aged adults is higher in the USA than in England. Using data on individuals aged 35-74 from six European countries, Canada and the USA, Wolf-Maier et al. (2003) found that prevalence of measured hypertension is higher in Europe. Crimmins et al. (2011) analysed data on adults aged 50+ from 11 European countries, England and the USA and found higher prevalence of self-reported hypertension in the USA. Higher rates of hypertension control and treatment were also found in the USA as compared to Western Europe (Wang et al. 2007; Wolf-Maier et al. 2004). None of these studies, however, used data from Ireland.

Prompted by the study by Banks et al. (2006), Savva et al. (2013) compared hypertension prevalence among adults aged 50+ across England, the USA and Ireland. Focusing on the American-Irish comparison, the authors found that while prevalence of self-reported hypertension is higher in the USA, prevalence of measured hypertension is higher in Ireland. As a formal investigation of why differences exist between the two countries was not carried out in this study, we attempt to fill this gap and have three aims.

Our first aim is to compare prevalence of diagnosed, measured, total and undiagnosed hypertension in the USA and Ireland, using recent data designed to provide comparable information across nationally representative samples of community-dwelling older populations in the two countries. Our second aim is to identify the most important factors leading to higher probability of diagnosed, measured, total and undiagnosed hypertension and to compare them across the two countries. Our third aim is to investigate whether and the extent to which crossnational differences in prevalence rates are explained by country differences in behavioural risk factors.

It is worth noting that the USA is one of the first countries to have embarked on national hypertension prevention and control programmes. The blood pressure education programme was established in 1972 (NHLBI 2014) and, since then, the USA has had one of the world's highest rates of hypertension awareness, treatment and control (Joffres et al. 2013; Egan et al. 2010). Cardiovascular disease remains, however, the single largest cause of death in the USA, accounting for $25 \%$ of all deaths in 2008 (Pagidipati and Gaziano 2013). In Ireland, the first cardiovascular health policy framework was introduced in 1999 and a second framework followed in 2010 (DOHC 1999, 2010). Despite improvements in death rates from cardiovascular disease, cardiovascular disease remains the single largest cause of death also in Ireland and accounted for $35 \%$ of all deaths in 2008 (DOHC 2010).

\section{Methods}

Data

For the USA, we use data from the Health and Retirement Survey (HRS), which is a biennial, population-representative, longitudinal study of the economic, health and social status of older Americans. It was launched in 1992 and initially recruited a sample of more than 12,000 non-institutionalised individuals aged $50+$. Respondents were recruited from a multistage area probability sample of households, with oversamples of Blacks, Hispanics, and residents from the state of Florida (Heeringa and Connor 1995; Juster and Suzman 1995). Respondents have since been followed up regularly with supplementary samples added at various waves.

For our analysis, we use the RAND HRS Data file, which is a user-friendly dataset based on the HRS data. It was prepared by the RAND Center for the Study of Aging with funding from the National Institute on Aging and the Social Security Administration. We use pooled data from the 2008 and 2010 interviews, when blood pressure measurements in randomly selected subsamples of the study population were collected (RAND HRS Data, Version L 2011a; RAND HRS Data, Version M 2011b).

For Ireland, we use data from The Irish Longitudinal Study on Ageing (TILDA), which is a biennial, populationrepresentative, longitudinal study of community-dwelling adults aged $50+$ residing in Ireland. The survey instrument was developed based on best international practice and harmonized with other large cohort studies of ageing, including HRS.

For our analysis, we use the first wave of TILDA, which was collected between October 2009 and July 2011. As explained in detail by Savva et al. (2013) and McGarrigle et al. (2014), the baseline sample was recruited from a clustered random sample of all households in Ireland. A total of 8,504 respondents $(8,175$ aged $50+$ and 329 younger spouses/partners of eligible individuals) completed a computer-assisted personal interview (CAPI) in their home. Information on various aspects of the respondents' lives, including the economic dimension, health aspects and the social domain, was collected.

Each respondent was also invited to travel to one of two health centres for a comprehensive health assessment, 
which was carried out by trained and qualified nurses. Respondents who were unable or unwilling to attend a health centre were offered a modified and partial assessment in their own home. The availability of home-based health assessments mitigated much of the potential selection bias associated with the need to travel to a health centre (Savva et al. 2013). Among other clinical parameters, cardiovascular measures were assessed and collated. A total of 5,898 respondents underwent an assessment: $85.4 \%$ in the health assessment and $15.6 \%$ in their own home. The overall response rate to the study was $62 \%$.

To ensure that hypertension prevalence rates are not confounded by age, we follow Banks et al. (2006) and limit the HRS-TILDA comparisons to those aged 57-64. Within this age span, the average age of both samples is identical. To ensure that differences between countries are not due to special issues that exist in the Black or Hispanic communities in the USA, we restrict the American sample to nonHispanic Whites (Banks et al. 2010; Langa et al. 2009). Although information on ethnic group is not collected in TILDA, respondents are asked about their country of birth. Around $92 \%$ of respondents were born in Ireland. Of those born elsewhere, 8 in 10 were born in Great Britain. We exclude from the TILDA sample respondents born in countries in which the population is predominantly nonWhite. The final sample sizes are 1,455 for TILDA and 1,938 for HRS.

Measures of hypertension prevalence

\section{Diagnosed}

Both HRS and TILDA collect data on individual selfreports of specific conditions with the general question: "Has the doctor ever told you have...?". We classify respondents as having "diagnosed hypertension" if they report hypertension among the conditions.

\section{Measured}

The key advantage in using data collected in HRS and TILDA is that blood pressure is measured in both surveys. Details on how blood pressure measurements are taken in the two surveys can be found elsewhere (Cronin et al. 2011; Crimmins et al. 2008). Briefly, in HRS, three measurements are taken by trained and qualified interviewers in the respondent's home. Measurements are taken 45-60 s apart with the respondent seated. An Omron HEM-780 intellisense automated blood pressure monitor with ComFit cuff is used. In TILDA, three separate readings are taken $1 \mathrm{~min}$ apart by trained and qualified nurses, either in a dedicated health assessment or in the respondent's home. The first two measurements are taken with the respondent seated and the third immediately after the respondent stands up. An Omron ${ }^{\mathrm{TM}}$ digital automatic blood pressure monitor with arm cuff (Model M10-IT) is used.

We create a binary variable for measured hypertension based on the mean value of the first and second readings. We classify respondents as hypertensive if: systolic blood pressure $(\mathrm{SBP}) \geq 140 \mathrm{mmHg}$ and/or diastolic blood pressure (DBP) $\geq 90 \mathrm{mmHg}$ (Mancia et al. 2007; NHLBI 2004).

\section{Total}

The total measure codes respondents as hypertensive if either they self-report to be hypertensive and/or have a blood pressure value above the diagnostic threshold, which is $\mathrm{SBP} \geq 140 \mathrm{mmHg}$ and/or $\mathrm{DBP} \geq 90 \mathrm{mmHg}$.

\section{Undiagnosed}

We classify respondents as having "undiagnosed hypertension" if they do not report having been told by a doctor that they have hypertension but are hypertensive according to the more comprehensive total prevalence measure. Put differently, the prevalence of undiagnosed hypertension is the fraction of total prevalence that is not diagnosed.

\section{Control variables}

We include age, gender and marital status as individual attributes. We use two measures of socioeconomic status: level of education and household income. To measure education, we use the International Standard Classification of Education (ISCED 97), which is a cross-national classification framework for harmonising educational programmes and qualifications (UNESCO 1997). We identify two groups: ISCED 0-3 (pre-primary, primary, lower secondary and upper secondary education); and ISCED 4+ (post-secondary non-tertiary or tertiary education).

Turning to income, for the USA we use data from RAND Income and Wealth Imputation file, produced by RAND Centre for the Study of Ageing (RAND 2011a, b). In this file, single imputation techniques are used to derive all components of income and household income is calculated by aggregating income across the respondent and her spouse if the respondent is married. For Ireland, we use information from a single comprehensive question about income across all household members. We correct household income by dividing it by the square root of the number of persons in the household (Buhmann et al. 1988; Avendano and Glymour 2008; Avendano et al. 2009; Huisman et al. 2003; Sarang et al. 2012). Finally, we assign individuals into income terciles, which are determined for each country and each wave separately. 
HRS and TILDA collect information on several healthrelated behaviours and respondents are asked whether they: have ever smoked tobacco; smoke at present; and drink alcohol. We identify three categories for smoking: current smoker, past smoker and never smoked; and two categories for alcohol consumption: current drinker and abstainer. Both surveys also collect objective measures of body mass index (BMI), which is the ratio of weight in kilograms to height in meters squared. Height and weight are measured during the enhanced face-to-face visit in HRS and during the health assessment in TILDA. We use BMI to identify whether respondents are obese $(\mathrm{BMI} \geq 30$ ), overweight $(\mathrm{BMI} \geq 25$ and $<30$ ) or underweight/normal $(\mathrm{BMI}<25)$. We separate obese individuals into three subgroups: class 1 $(\geq 30$ and $<35)$, class $2(\geq 35$ and $<40)$ and class $3(\geq 40)$.

We also include a variable meant to capture the extent of contact with the medical system. For HRS, we include a dichotomous variable equal to one if the respondent has had outpatient surgery, has stayed in an hospital overnight or has talked to a medical doctor about her health, including emergency room, clinic visits or house calls, at least once in the 2 years prior to the interview. For TILDA, we include a dichotomous variable equal to one if the respondent has visited a general practitioner, a hospital emergency department, a hospital as outpatient or a hospital overnight at least once in the year prior to the interview.

Although other risk factors might be important, due to the comparative nature of our research, we only use those comparably measured in both countries.

Statistical methods

We use unweighted probit models of determinants of diagnosed, measured, total and undiagnosed hypertension prevalence. We use STATA 12 to perform the analyses (StataCorp 2011) and set a prior level of significance at $p<0.05$. For ease of interpretation, we convert parameter estimates to estimates of average marginal effects (AMEs). Marginal effects are a popular means by which the effects of variables in nonlinear models can be made more intuitively meaningful (Williams 2012). To illustrate, the AME of a categorical variable is the mean change in the predicted probability that the outcome is equal to one as the categorical variable changes from 0 to 1 , holding all other covariates at their observed values.

\section{Results}

Baseline characteristics

Table 1 lists the prevalence of diagnosed, measured, total and undiagnosed hypertension. The results of statistical tests for country differences are also displayed.
Table 1 Hypertension prevalence rates and hypertension risk factors in the USA (2008/2010) and Ireland (2009/2011): 57-64 years old

\begin{tabular}{|c|c|c|}
\hline & USA & Ireland \\
\hline \multicolumn{3}{|l|}{ Hypertension prevalence $^{\mathrm{a}}$} \\
\hline Diagnosed & 48.6 & $32.4 * * *$ \\
\hline Measured & 30.0 & $39.5 * * *$ \\
\hline Total & 60.6 & $55.2 * *$ \\
\hline Undiagnosed & 19.7 & $41.2 * * *$ \\
\hline \multicolumn{3}{|l|}{ Individual characteristics } \\
\hline Age, mean & 60.4 & 60.4 \\
\hline Male & 42.8 & 45.9 \\
\hline Married & 74.4 & 76.2 \\
\hline \multicolumn{3}{|l|}{ Behavioural health } \\
\hline \multicolumn{3}{|l|}{ Smoking } \\
\hline Never smoked & 45.0 & 45.4 \\
\hline Past smoker & 38.6 & 39.2 \\
\hline Current smoker & 16.3 & 15.4 \\
\hline \multicolumn{3}{|l|}{ Drinking } \\
\hline Current drinker & 64.5 & $82.7 * * *$ \\
\hline \multicolumn{3}{|l|}{ BMI category } \\
\hline Under/normal weight & 20.8 & 21.2 \\
\hline Overweight & 35.5 & $44.4 * * *$ \\
\hline Obese class 1 & 26.2 & 24.7 \\
\hline Obese class 2 & 11.5 & $7.1 * * *$ \\
\hline Obese class 3 & 5.9 & $2.6 * * *$ \\
\hline $1+$ contact with medical system & 95.2 & $88.4 * * *$ \\
\hline \multicolumn{3}{|l|}{ Socioeconomic gradient } \\
\hline \multicolumn{3}{|l|}{ Education } \\
\hline ISCED 4+ & 40.6 & $35.1 * *$ \\
\hline \multicolumn{3}{|l|}{ Adjusted household income } \\
\hline First tercile & 34.2 & 34.2 \\
\hline Second tercile & 33.1 & 34.5 \\
\hline Third tercile & 32.7 & 31.3 \\
\hline$N$ & 1,938 & 1,455 \\
\hline
\end{tabular}

BMI body mass index, ISCED International Standard Classification of Education

$* p<0.05, * * p<0.01, * * * p<0.001$

a Diagnosed prevalence, whether a doctor told the respondent that they were hypertensive; measured prevalence, SBP $\geq 140 \mathrm{mmHg}$ and/or $\mathrm{DBP} \geq 90 \mathrm{mmHg}$; total prevalence, either self-report or measured; undiagnosed prevalence, the fraction of total prevalence that is not diagnosed

Prevalence of diagnosed hypertension is higher in the USA. Prevalence of measured and undiagnosed hypertension is higher in Ireland. Total prevalence is higher in the USA.

Table 1 also documents levels in prominent hypertension risk factors. The most striking differences are that class 2 and class 3 obesity are significantly higher in the USA and drinking is higher in Ireland. 


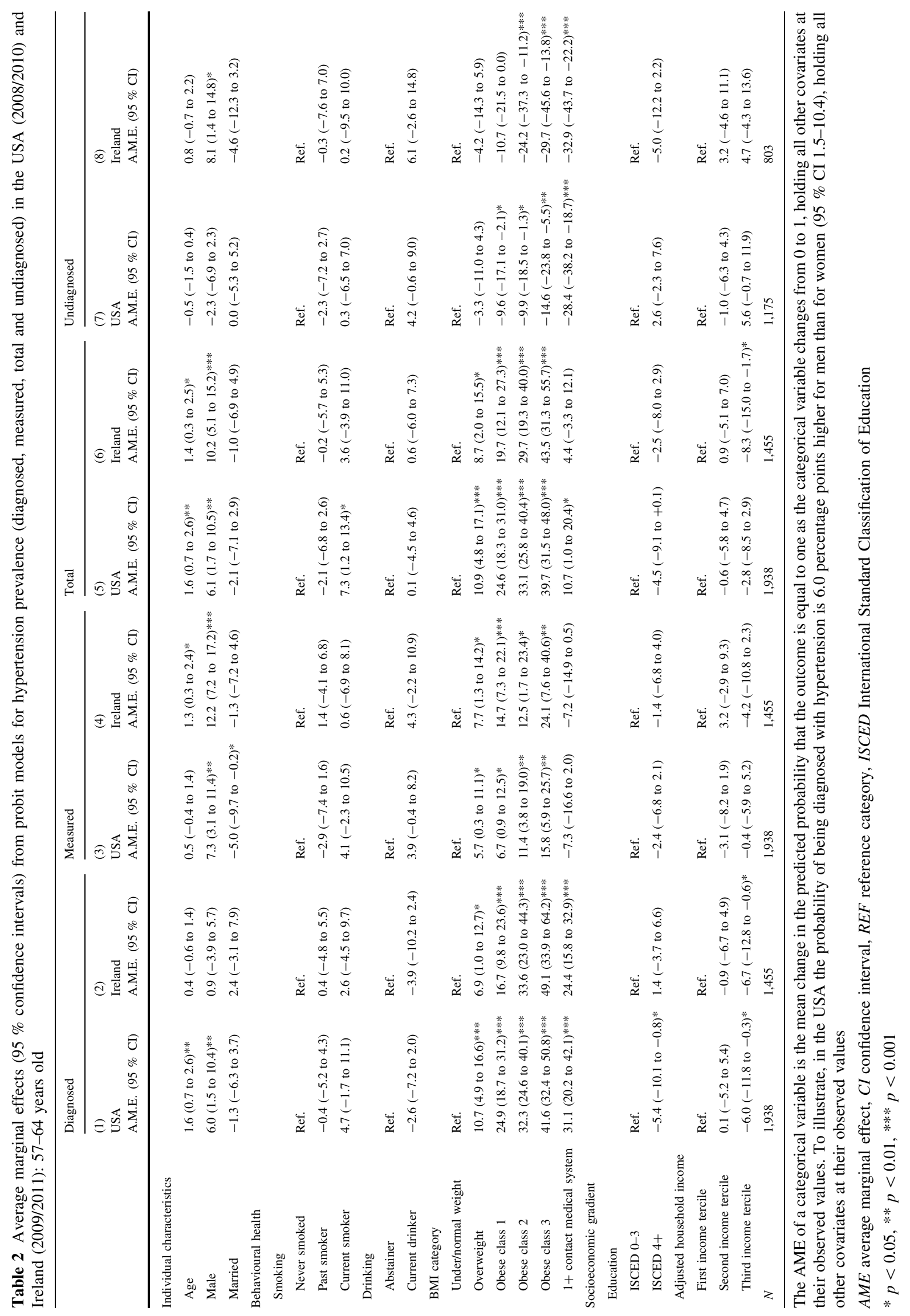


Predictors of hypertension prevalence and between-country comparisons

Table 2 lists estimates of probit models for diagnosed, measured, total and undiagnosed hypertension prevalence in the USA and Ireland. The purpose of these models is twofold. The first is to identify the most important factors leading to higher probability of diagnosed, measured, total and undiagnosed hypertension, and the second is to compare them across the two countries.

In both countries, obesity and contact with the medical system are positively associated with the probability of hypertension diagnosis (Columns 1 and 2). The probability of having high blood pressure objectively is higher for individuals who are men and obese (Columns 3 and 4). The probability of being hypertensive based on the more comprehensive total measure is higher for individuals who are older, men and overweight or obese, with the estimated obesity impact being much larger than overweight (Columns 5 and 6). The most important predictors of undiagnosed hypertension are absence of contact with the medical system in the recent past and normal weight (Columns 7 and 8).

A number of differences in the sign or significance of hypertension risk factors across the two countries also emerge. Most notably, older adults and men are more likely to be diagnosed in USA, but not in Ireland. Men are more likely to be undiagnosed in Ireland, but not in the USA. Also, married adults are less likely to have high blood pressure objectively in the USA, but not in Ireland.

Do differences in risk factors explain between-country differences in prevalence rates?

Table 1 showed that prevalence rates of diagnosed and undiagnosed hypertension in the USA are 48.6 and $19.7 \%$, respectively. These compare to 32.4 and $41.2 \%$ in Ireland. We now use the models of Table 2 to simulate what the prevalence rates of diagnosed and undiagnosed hypertension in Ireland would be if Irish individuals had the same level of behavioural risk factors (drinking, smoking, obesity and contact with medical system) as the average American in this age group. In this simulation, we find that risk-factor adjusted prevalence rates of diagnosed and undiagnosed hypertension in Ireland would be 36.2 and $36.3 \%$, respectively.

By comparing the unadjusted and adjusted prevalence rates, we can understand how much of the between-country difference in hypertension prevalence is due to different levels of behavioural risk factors. This comparison shows that only around one-fourth $[(36.2-32.4 \%) /(48.6-32.4 \%)]$ of the difference in prevalence of diagnosed hypertension is due to differences in this subset of behavioural risk factors. Similarly, only less than one-fourth of the difference in prevalence of undiagnosed hypertension is explained by differences in risk factors.

\section{Discussion}

Cross-national comparisons of health outcomes are rising in importance as a method of addressing key questions in international public health and of gaining insight into the complex determinants of health. In this paper, we took advantage of the comparability built into the design of the Health and Retirement Study and The Irish Longitudinal Study on Ageing to compare prevalence of diagnosed, measured, total and undiagnosed hypertension among late middle-aged adults across the USA and Ireland. We also identified and compared the most important predictors of hypertension and investigated how much of the crossnational differences in prevalence rates are explained by country differences in behavioural risk factors. It is worth noting that cardiovascular disease remains the single largest cause of death in both Ireland and the USA. Both countries have also adopted hypertension prevention strategies, although these have been in place for much longer in the USA.

Our results show that, based on the more comprehensive total measure that combines diagnosed and measured hypertension, prevalence of hypertension is higher in the USA. There exist, however, remarkable differences in prevalence of diagnosed, measured and undiagnosed hypertension. While prevalence of diagnosed hypertension is higher in the USA, prevalence of measured and undiagnosed hypertension is higher in Ireland. Our findings are in line with those of Wolf-Maier et al. (2003), Crimmins et al. (2011) and Savva et al. (2013), as they confirm higher prevalence of self-reported hypertension in the USA as opposed to higher rates of measured hypertension in Ireland/Europe.

Turning to the predictors of hypertension prevalence, three results stand out.

First, in both countries obesity is positively associated with diagnosed, measured and total hypertension and negatively associated with undiagnosed hypertension.

Second, in both countries, 'contact with the medical system' is positively associated with the probability of being diagnosed and negatively associated with the probability of being undiagnosed. The interpretation of this finding is, however, problematic. It might be that more frequent visits provide added opportunity for physicians to detect undiagnosed hypertension, or the causality might be reversed, and those who have already been diagnosed make more visits to refill prescriptions or monitor treatment effectiveness. 
Third, in both countries men are more likely to have high blood pressure objectively and to be hypertensive when the most comprehensive definition of hypertension is used. Compared to their female counterparts, however, men are more likely to be diagnosed in the USA and to be undiagnosed in Ireland.

Turning finally to the role of behavioural risk factors in explaining cross-national differences in prevalence rates, we find that, even if the Irish had the same level of behavioural risk factors as the average American in this age group, this would explain around one quarter of the difference in prevalence of diagnosed and undiagnosed hypertension.

The question remains as to why such large discrepancies exist in prevalence rates across the two countries.

One hypothesis behind higher rates of diagnosed hypertension in the USA is that protocols and thresholds for hypertension diagnosis may differ between the two countries so that a similarly ill patient is diagnosed with hypertension in the USA but not in Ireland. For guidance on blood pressure measurement and hypertension diagnosis, the USA follows the Seventh Report of the Joint National Committee on Prevention, Detection, Evaluation, and Treatment of High Blood Pressure (NHLBI 2004) whereas Ireland follows the European Society of Cardiology (ESC) 2007 Guidelines for Cardiovascular Disease Prevention (Mancia et al. 2007). These guidelines are, however, generally similar. Another hypothesis behind higher rates of diagnosed hypertension in the USA is that the language by which physicians communicate health problems to their patients may differ across the two countries. Also, although we control for 'quantity' of contacts with the medical system in the empirical model, we have no information on the 'quality' of these contacts and, again, this may differ among the two countries. Overall differences in the clinical systems, community programmes and environmental and policy support are likely to be important factors behind the cross-national differences in hypertension prevalence rates.

As rates of measured hypertension are considerably higher in Ireland, we reflect on whether differences in protocols for blood pressure measurements in HRS and TILDA may be responsible. In particular, as for most TILDA respondents blood pressure is measured in a dedicated health assessment, higher rates of measured hypertension may be explained by the so-called "white coat effect', which is higher than normal blood pressure values when measured in a medical environment (Mancia et al. 2007; Pickering et al. 1999). We investigate this hypothesis by comparing blood pressure readings for TILDA respondents who underwent a health assessment in their own home as opposed to a dedicated health assessment. We find no evidence that, ceteris paribus, significant differences in blood pressure readings exist among the two groups.
Our findings should be considered in the context of several limitations.

First, as all data analyses are cross-sectional, we investigate associations and are unable to confidently identify the causes of differences in prevalence rates among the two countries.

Second, the results on prevalence of measured hypertension need to be interpreted with caution, for a number of reasons. In both studies, prevalence of measured hypertension is determined based on measurements carried out on a single day and not on multiple measurements taken on two or more occasions over a period of time as suggested by the ESC Guidelines for Cardiovascular Disease Prevention (Mancia et al. 2007). While important, this limitation applies to all studies based on data from HRS and its 'sister' studies and to a number of other international studies (for example, see Kaur et al. 2012; Laaser et al. 2012; Ploubidis et al. 2013). Also, as the third blood pressure measurement in TILDA is taken after the respondent stands up, we use the average of the first two measurements, which are taken with the respondent seated in both studies. Because of the so-called 'white collar effect', the use of the first two readings might lead to a possible overestimation of hypertension prevalence in both countries. Finally, we do not distinguish between respondents who are on antihypertensive medication and are untreated. This is because a comprehensive list of prescription medications obtained by direct observation of the interviewer is collated in TILDA, but not in HRS.

Third, the list of comparably measured behavioural risk factors and individual attributes is incomplete and a fuller set may offer additional explanatory power. For example, we do not include dietary patterns, as they are not measured in either study. We cannot exclude that a greater proportion of the cross-national differential in prevalence rates could have been explained had a more extensive list of behavioural risk factors been used.

Fourth, we do not take into account past differences in risk factors, but these may be partially underlying observed differences in prevalence rates. For example, only in recent years the obesity epidemic has hit Ireland where prevalence of obesity rose from 11 to $25 \%$ between 1998 and 2008, compared to an increase from 20 to $32 \%$ in the USA in the same period (WHO 2012).

Fifth, some differences exist in the phrasing of the questions posed to respondents. To illustrate, retrospective information on frequency of contact with the medical system is collected for the year prior to the interview in TILDA, compared to 2 years, which is since the last interview, in HRS.

Despite these limitations, it is noteworthy that this study is unique in using recent data designed to provide comparable information on health outcomes across nationally 
representative samples of community-dwelling older populations in Ireland and the USA. As international comparability with HRS was an important consideration in the design of TILDA, the methods used in the two studies are remarkably similar.

In conclusion, our results indicate that prevalence of measured and undiagnosed hypertension among late middle-aged adults is considerably higher in Ireland than in the USA and that much of this difference is not explained by conventional risk factors. Our results suggest that a greater focus on prevention of high blood pressure is necessary in Ireland.

Acknowledgments TILDA is funded by Atlantic Philanthropies, the Irish Government Department of Health and Children and Irish Life and Permanent plc. HRS is funded by The National Institute on Aging. The RAND HRS data files used in the analysis of this paper were prepared by the RAND Center for the Study of Aging with funding from the National Institute on Aging and the Social Security Administration.

\section{References}

Avendano M, Glymour MM (2008) Stroke disparities in older Americans. Is wealth a more powerful indicator of risk than income and education? Stroke 39:1533-1540

Avendano M, Glymour MM, Banks J, Mackenbach JP (2009) Health disadvantage in US adults aged 50 to 74 years: a comparison of the health of rich and poor Americans with that of Europeans. Am J Public Health 99(3):150-158

Banks J, Marmot M, Oldfield Z, Smith JP (2006) Disease and disadvantage in the United States and in England. JAMA 295(17):2037-2045

Banks J, Muriel A, Smith JP (2010) Disease prevalence, disease incidence, and mortality in the United States and England. Demography 47 Supplement: S211-S231

Buhmann B, Rainwater L, Schmaus G, Smeeding TM (1988) Equivalence scales, well-being, inequality and poverty; sensitivity estimates across ten countries using the Luxembourg income study (LIS) data base. Rev Income Wealth 34(2):115-142

Crimmins EM, Guyer H, Langa K, Ofstedal M, Wallace R, Weir D (2008) Documentation of physical measures, anthropometrics and blood pressure in the Health and Retirement Study. Survey Research Center. University of Michigan, Ann Arbor

Crimmins EM, Kim JK, Solé-Auró A (2011) Gender differences in health: results from SHARE, ELSA and HRS. Eur J Public Health 21(1):81-91

Cronin H, O'Regan C, Kenny RA (2011) Physical and behavioural health of older Irish adults. In: Barrett A, Savva G, Timonen V, Kenny RA (eds) Fifty plus in Ireland 2011: first results from The Irish Longitudinal Study on Ageing. Trinity College, Dublin, pp 73-154

Department of Health and Children (DOHC) (1999) Building healthier hearts Introduction to the report of the cardiovascular health strategy group. Government Publications, Dublin

Department of Health and Children (DOHC) (2010) Changing cardiovascular health national cardiovascular health policy 2010-2019. Government Publications, Dublin

Egan BM, Zhao Y, Axon RN (2010) US trends in prevalence, awareness, treatment, and control of hypertension, 1988-2008. JAMA 303:2043-2050
Heeringa SG, Connor J (1995) Technical description of the health and retirement study sample design. Survey Research Center. University of Michigan, Ann Arbor

Huisman M, Kunst AE, Mackenbach JP (2003) Socioeconomic inequalities in morbidity among the elderly: a European overview. Soc Sci Med 57:861-873

Joffres M, Falaschetti E, Gillespie C, Robitaille C, Loustalot F et al (2013) Hypertension prevalence, awareness, treatment and control in national surveys from England, the USA and Canada, and correlation with stroke and ischaemic heart disease mortality: a cross-sectional study. BMJ Open 3:e003423. doi:10.1136/ bmjopen-2013-003423

Juster FT, Suzman R (1995) An overview of the health and retirement study. J Hum Res 30:S7-S56

Kaur P, Rao SR, Radhakrishnan E, Rajasekar D, Gupte MD (2012) Prevalence, awareness, treatment, control and risk factors for hypertension in a rural population in South India. Int J Public Health 57:87-94

Kearney PM, Whelton M, Reynolds K, Muntner P, Whelton PK, He J (2005) Global burden of hypertension: analysis of worldwide data. Lancet 365:217-223

Laaser U, Breckenkamp J, Bjegovic V (2012) Treatment of hypertension in Germany: is there a social gradient? Int J Public Health 57:185-191

Langa KM, Llewellyn DJ, Lang IA, Weir D, Wallace RC et al (2009) Cognitive health among older adults in the United States and in England. BMC Geriatr. doi:10.1186/1471-2318-9-23

Mancia G, De Backer G, Dominiczak A, Cifkova R, Fagard R et al (2007) Guidelines for the management of arterial hypertension: the task force for the management of arterial hypertension of the European Society of Hypertension (ESH) and of the European Society of Cardiology (ESC). Eur Heart J 28:1462-1536

McGarrigle CA, Cronin H, Kenny RA (2014) The impact of being the intermediate caring generation and intergenerational transfers on self-reported health of women in Ireland. Int J Public Health 59(2):301-308

NHLBI (2004) The seventh report of the Joint National Committee on prevention, detection, evaluation, and treatment of high blood pressure. NIH Publication No. 04-5230

NHLBI (2014) National high blood pressure education program. http://www.nhlbi.nih.gov/about/nhbpep/. Accessed 9 May 2014

Pagidipati NJ, Gaziano TA (2013) Estimating deaths from cardiovascular disease: a review of global methodologies of mortality measurement. Circulation 127:749-756

Pickering TG, Coats A, Mallion JM, Mancia G, Verdecchia P (1999) Blood pressure monitoring. Task force V: white-coat hypertension. Blood Press Monit 4:333-341

Ploubidis GB, Mathenge W, De Stavola B, Grundy E, Foster A, Kuper H (2013) Socioeconomic position and later life prevalence of hypertension, diabetes and visual impairment in Nakuru, Kenya. Int J Public Health 58:133-141

RAND (2011a) RAND HRS 2008 Income and wealth imputations. Produced by the RAND Center for the Study of Aging, with funding from the National Institute on Aging and the Social Security Administration. Santa Monica, CA

RAND (2011b) RAND HRS 2010 Income and wealth imputations. Produced by the RAND Center for the Study of Aging, with funding from the National Institute on Aging and the Social Security Administration. Santa Monica, CA

RAND HRS Data, Version L (2011a) Produced by the RAND Center for the Study of Aging, with funding from the National Institute on Aging and the Social Security Administration. Santa Monica, CA

RAND HRS Data, Version M (2011b) Produced by the RAND Center for the Study of Aging, with funding from the National Institute on Aging and the Social Security Administration. Santa Monica, CA 
Sarang K, Sargent-Cox KA, French DJ, Kendig H, Anstey KJ (2012) Cross-national insights into the relationship between wealth and wellbeing: a comparison between Australia, the United States of America and South Korea. Ageing Soc 32:41-59

Savva GM, Maty SC, Setti A, Feeney J (2013) Cognitive and physical health of the older populations of England, the United States, and Ireland: international comparability of The Irish Longitudinal Study on Ageing. JAGS 61(s2):S291-S298

StataCorp (2011) Stata: Release 12. Statistical Software. StataCorp LP, College Station

UNESCO (1997) International Standard Classification of Education. UNESCO, Paris

Wang YR, Alexander GC, Stafford RS (2007) Outpatient hypertension treatment, treatment intensification, and control in Western Europe and the United States. Arch Intern Med 67(2):141-147
WHO (2012) Global Health Observatory (GHO). Country statistics. http://www.who.int/gho/countries/en/index.html. Accessed 9 May 2014

WHO (2013) A global brief on hypertension. Silent killer, global public health crisis. Report no.: WHO/DCO/WHD/2013.2

Williams R (2012) Using the margins command to estimate and interpret adjusted predictions and marginal effects. Stata J 12(2):308-331

Wolf-Maier K, Cooper RS, Banegas JR, Giampaoli S, Hense HW et al (2003) Hypertension prevalence and blood pressure levels in 6 European countries, Canada, and the United States. JAMA 289:2363-2369

Wolf-Maier K, Cooper RS, Kramer H, Banegas JR, Giampaoli S et al (2004) Hypertension treatment and control in five European countries, Canada, and the United States. Hypertension 43:10-17 